\title{
Clinical outcomes of positive resection margin after endoscopic mucosal resection of early colon cancers
}

\author{
Junseok Park ${ }^{1}$, Hyun Gun Kim ${ }^{1}$, Shin Ok Jeong ${ }^{1}$, Hoon gil Jo ${ }^{1}$, Hyo Yeop Song ${ }^{1}$, Jeeyeon Kim ${ }^{1}$, Seri Ryu ${ }^{1}$, \\ Youngyun Cho ${ }^{1}$, Hyun Jin Youn ${ }^{1}$, Seong Ran Jeon ${ }^{1}$, Jin-Oh Kim ${ }^{1}$, Bong Min Ko ${ }^{1}$, Yoon Mi Jeen ${ }^{2}$, So-Young Jin ${ }^{2}$ \\ Departments of ${ }^{1}$ Internal Medicine and ${ }^{2}$ Pathology, Soonchunhyang University College of Medicine, Seoul, Korea
}

Background/Aims: When determining the subsequent management after endoscopic resection of the early colon cancer (ECC), various factors including the margin status should be considered. This study assessed the subsequent management and outcomes of ECCs according to margin status. Methods: We examined the data of 223 ECCs treated by endoscopic mucosal resection (EMR) from 215 patients during 2004 to 2014, and all patients were followed-up at least for 2 years. Results: According to histological analyses, the margin statuses of all lesions after EMR were as follows: 138 cases (61.9\%) were negative, 65 cases (29.1\%) were positive for dysplastic cells on the resection margins, and 20 cases (8.9\%) were uncertain. The decision regarding subsequent management was affected not only by pathologic outcomes but also by the endoscopist's opinion on whether complete resection was obtained. Surgery was preferred if the lesion extended to the submucosa (odds ratio [OR], 25.46; 95\% confidence interval [CI], 7.09-91.42), the endoscopic resection was presumed incomplete (OR, 15.55; 95\% CI, 4.28-56.56), or the lymph system was invaded (OR, 13.69; 95\% CI, 1.76-106.57). Fourteen patients (6.2\%) had residual or recurrent malignancies at the site of the previous ECC resection and were significantly associated with presumed incomplete endoscopic resection (OR, 4.59; 95\% CI, 1.21-17.39) and submucosal invasion (OR, 5.14; 95\% CI, 1.18-22.34). Conclusions: Subsequent surgery was associated with submucosa invasion, lymphatic invasion, and cancer-positive margins. Presumed completeness of the resection may be helpful for guiding the subsequent management of patients who undergo endoscopic resection of ECC. (Intest Res 2019;17:516-526)

Key Words: Early colon cancer; Endosocpic mucosal resection; Margins of excision

\section{INTRODUCTION}

Early colon cancer (ECC) is defined as cancer that has invaded the mucosal and submucosal layers and can be cured if it is endoscopically or surgically treated depending on the characteristics of the lesion. Many characteristics of ECCs are relevant for selecting the subsequent management and are based on histological features and procedural considerations. Previous studies have shown that deep submucosal invasion, vas-

Received December 20, 2018. Revised April 16, 2019. Accepted April 22, 2019. Correspondence to Hyun Gun Kim, Department of Internal Medicine, Soonchunhyang University College of Medicine, 59 Daesagwan-ro, Yongsangu, Seoul 04401, Korea. Tel: +82-2-710-3072, Fax: +82-2-709-9868, E-mail: medgun@schmc.ac.kr cular and lymphatic invasion, poor histological differentiation, and tumor budding in histological sections are strongly associated with lymph node metastasis of ECCs. ${ }^{1-6}$ If lesions with these unfavorable factors are initially resected endoscopically, the lesion should be subsequently treated surgically to reduce the risk of lymph node metastasis or early recurrence. Previous studies have revealed an association between endoscopic margin status and the risks for residual, recurrent, and advanced disease after endoscopic resection. ${ }^{7-14}$ As resection margin status is included in the initial pathology report, it directly influences clinical decision making. However, a positive resection margin does not always imply that there is remnant disease, and other variables should be considered, such as the resection technique, cautery artifacts, and problems with the patho- 
logical interpretation of the resected specimen. The rate of residual cancer diagnosed after additional surgery on a positive resection margin varies based on the evidence. A recent Korean study reported a $6.1 \%$ residual cancer rate after subsequent surgery for positive dysplastic cells on the endoscopic resection margin and $7.7 \%$ recurrent cancer in cases without subsequent surgery during surveillance. ${ }^{15}$ Another study found $15.3 \%$ residual cancer after additional surgery on lesions that received noncurative endoscopic resection. ${ }^{16}$ By contrast, a case with a negative resection margin could also have residual cancer or recurrent cancer during surveillance. There is limited evidence on the clinical outcomes of patients after subsequent management according to endoscopic resection margin status. In this study, we evaluated the clinical outcomes of ECCs by subsequent management according to margin status and factors af- fecting residual cancer after endoscopic mucosal resection (EMR).

\section{METHODS}

\section{Patients and Data Collection}

This retrospective study evaluated medical records from Soonchunhyang University Hospital (Seoul, Korea). Consecutive patients were considered eligible if they had ECC, were treated by EMR between January 2004 and December 2014, and were followed-up > 2 years. Our Institutional Review Board (IRB No. 2016-09-024) approved this protocol and data extraction from the patients' clinical images, pathology reports, and other relevant data sources. The informed consent was waived.

The patients' sex and age, lesion size and location, a written
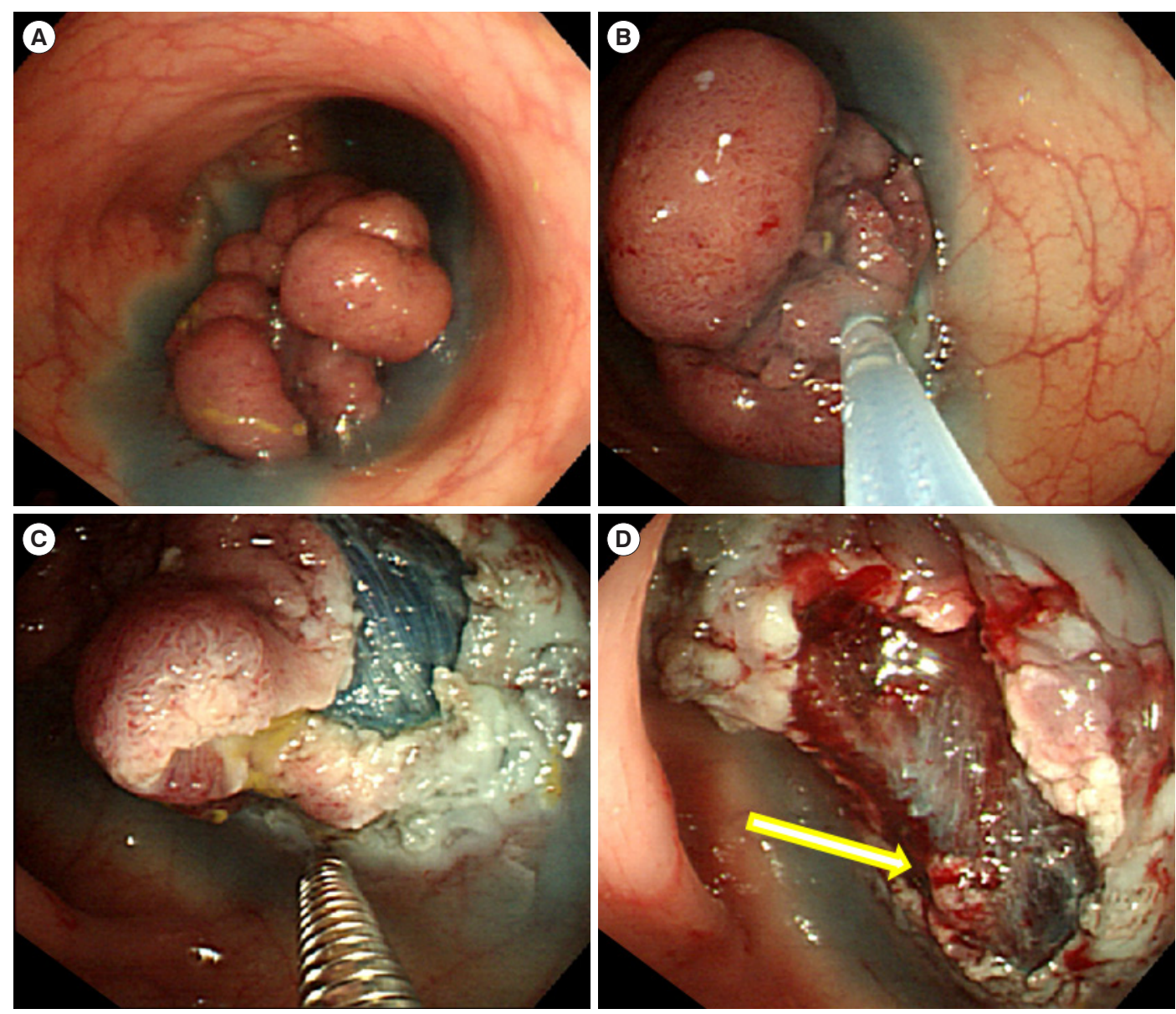

Fig. 1. Endoscopically presumed incomplete resection. (A) A nodular, mixed, laterally spreading tumor was identified after submucosal injection of a saline and indigo carmine solution. (B) Endoscopic piecemeal mucosal resection (EPMR) was performed using a conventional snare. (C) Additional injection of saline and indigo carmine during EPMR. (D) A section of the tumor removed using EPMR. The arrow indicates tissue that was torn during the procedure and was considered a possible remnant tumor island. It was difficult to determine whether or not the tumor had been completely removed even at the lateral resection margin. 
detailed description of the endoscopic procedure, and the appearance of the lesion immediately after EMR, which the endoscopist recorded as an endoscopic presumption for complete removal (or not), were extracted from the procedure reports. Clear resection margins without suspected remnant tumor tissue, based on the available images and endoscopic reports, were categorized as presumed complete resection (PCR). In contrast, cases with suspected remnant tumor tissue based on photographic records, or with incomplete resection noted on procedure reports, were categorized as presumed incomplete resection (PIR) (Fig. 1). Cases with mucosal changes that were obscured by diathermic injury, or with doubtfully clear margins, were classified as having an uncertain resection status.

Lesion locations were classified as: the right side of the colon (cecum and ascending colon), the left side of the colon (sigmoid and descending colon), the transverse colon, and the rectum. The endoscopic appearance of the lesions was categorized according to the Japanese classification system: pedunculated, protruded, flat or depressed, and laterally spreading. ${ }^{17}$ Procedures that successfully removed the entire lesion in one attempt were defined as en bloc, and all other procedures were classified as piecemeal. All resected lesions were evaluated by certified pathologists, who recorded the histological properties of the lesions. The definition of ECC was a carcinoma with invasion no deeper than the submucosa (SM) and included intramucosal cancer and carcinoma in situ. Resection margins were evaluated in the lateral and vertical directions and were considered positive if there were identifiable malignant or premalignant cells at the margins. They were categorized as adenoma, either with or without high-grade dysplasia, and as adenocarcinoma. Uncertain resection margins were defined as cases with a pathology report that described unclear margins. Negative margins were defined as cases without dysplastic cells on the resection margin.

The patients were categorized according to whether they underwent subsequent surgery or surveillance colonoscopy. Outcomes in these groups were analyzed according to the histological results, the presence of remnant tumor tissue, malignancy, and recurrence during the follow-up period.

We determined the clinical outcomes among patients with positive margins after ECC resection according to their subsequent management and analyzed the variables that were associated with residual disease and/or that influenced subsequent clinical decision-making.

\section{Statistical Analyses}

Statistical analyses were performed using Student $t$-test, Pearson chi-square test, and logistic regression analyses, as appropriate. Differences were considered significant at a $P$-value $<0.05$. All analyses were performed using SPSS software version 22.0 (IBM Corp., Armonk, NY, USA). Continuous variables are reported as mean and standard deviation or median and range. Categorical variables are reported as a number and proportions.

\section{RESULTS}

\section{Patients and Endoscopic Procedures}

Ten experienced endoscopists removed all lesions that exhibited neoplastic features during endoscopy between 2004 and 2014. All resected lesions were evaluated by 3 pathologists. A total of 223 lesions from 215 patients ( 137 men and 78 women; mean age, $59.3 \pm 9.2$ years) were diagnosed with ECC and underwent surveillance colonoscopy with or without subsequent surgery. During a median follow-up period of 47 months (range, 12-129 months), the patients underwent endoscopy an average of $4.0 \pm 2.2$ times (Table 1 ).

Among the 223 lesions, 80 (35.9\%) were from the left colon, $73(32.7 \%)$ were from the rectum, 39 (17.5\%) were from the right colon, and 31 (13.9\%) were from the transverse colon. Most lesions were removed en bloc via EMR (180, 80.7\%), and 43 lesions (19.3\%) were removed via piecemeal EMR. Based on the procedural report and accompanying images, 204 lesions (91.5\%) were PCRs, and 17 (7.6\%) were PIRs. Two lesions $(0.9 \%)$ had insufficient information and were assigned an unclear resection status.

\section{Histological Findings}

Among the 223 lesions, 119 (53.4\%) were well-differentiated, 48 (21.5\%) were moderately differentiated, and $1(0.4 \%)$ was a poorly differentiated adenocarcinoma. Malignant cells were confined to the mucosa in 167 lesions (74.9\%), and SM invasion was identified in 56 lesions (25.1\%). Lymphatic invasion was noted in 9 cases with SM invasion (4.0\%) (Table 1).

A histological review of the resection margins revealed that 138 lesions (61.9\%) had negative margins, and 65 (29.1\%) had positive margins, including $56(25.1 \%)$ with a positive lateral margin, $3(1.3 \%)$ with a positive vertical margin, and $6(2.7 \%)$ with both margins considered positive. Adenoma with lowgrade dysplasia was the identified cell type in 42 cases (18.8\%), high-grade dysplasia was identified in 4 cases (1.8\%), and defi- 
Table 1. General Characteristics of the Patients and Lesions

\begin{tabular}{|c|c|}
\hline Characteristic & No. $(\%)$ \\
\hline \multicolumn{2}{|l|}{ Patient $(n=215)$} \\
\hline \multicolumn{2}{|l|}{ Sex } \\
\hline Male & $137(63.7)$ \\
\hline Female & $78(36.3)$ \\
\hline \multicolumn{2}{|l|}{ Age at diagnosis (yr) } \\
\hline Mean \pm SD & $59.3 \pm 9.2$ \\
\hline \multicolumn{2}{|l|}{ Following management } \\
\hline Surveillance endoscopy & $183(85.1)$ \\
\hline Subsequent surgery & $32(14.8)$ \\
\hline \multicolumn{2}{|c|}{ First surveillance endoscopy (day, after the resection of ECC) } \\
\hline Mean \pm SD & $296.8 \pm 426.3$ \\
\hline Median (range) & $153(22-3,220)$ \\
\hline \multicolumn{2}{|l|}{ Follow-up duration (mon) } \\
\hline Mean \pm SD & $51.5 \pm 30.2$ \\
\hline Median (range) & $47(12-129)$ \\
\hline \multicolumn{2}{|c|}{ Follow-up endoscopy (number of times) } \\
\hline Mean \pm SD & $4.0 \pm 2.2$ \\
\hline Median (range) & $4(1-11)$ \\
\hline \multicolumn{2}{|l|}{ Lesion $(n=223)$} \\
\hline \multicolumn{2}{|l|}{ Size $(m m)$} \\
\hline Mean \pm SD & $18.7 \pm 15.0$ \\
\hline \multicolumn{2}{|l|}{ Shape } \\
\hline Pedunculated & $71(31.8)$ \\
\hline Protruded & $51(22.9)$ \\
\hline Flat & $29(13.0)$ \\
\hline Depressed & $3(1.3)$ \\
\hline LST, G-M & 31 (13.9) \\
\hline LST, G-H & $22(9.9)$ \\
\hline LST, NG-F & $13(5.8)$ \\
\hline LST, NG-PD & $3(1.3)$ \\
\hline \multicolumn{2}{|l|}{ Location } \\
\hline Right colon & $39(17.5)$ \\
\hline Transverse colon & $31(13.9)$ \\
\hline Left colon & $80(35.9)$ \\
\hline Rectum & 73 (32.7) \\
\hline \multicolumn{2}{|l|}{ Resection method } \\
\hline EMR & $180(80.7)$ \\
\hline EPMR & 43 (19.3) \\
\hline \multicolumn{2}{|l|}{ Histological differentiation } \\
\hline Carcinoma in situ & $55(24.7)$ \\
\hline Adenocarcinoma & 0 \\
\hline Well differentiated & $119(52.9)$ \\
\hline Moderately differentiated & $48(21.5)$ \\
\hline Poorly differentiated & $1(0.4)$ \\
\hline
\end{tabular}

(Continued to the next)
Table 1. Continued

\begin{tabular}{|c|c|}
\hline Characteristic & No. (\%) \\
\hline \multicolumn{2}{|l|}{ Invasion depth } \\
\hline Mucosa & $167(74.9)$ \\
\hline Submucosa & $56(25.1)$ \\
\hline \multicolumn{2}{|l|}{ Lymphatic invasion } \\
\hline Positive & $9(4.0)$ \\
\hline Negative & $214(96.0)$ \\
\hline \multicolumn{2}{|l|}{ Resection margin } \\
\hline Negative & $138(61.9)$ \\
\hline Positive & $65(29.1)$ \\
\hline \multicolumn{2}{|l|}{ Direction } \\
\hline Lateral & $56(25.1)$ \\
\hline Vertical & $3(1.3)$ \\
\hline Both & $6(2.7)$ \\
\hline \multicolumn{2}{|l|}{ Histology } \\
\hline Adenoma, low-grade dysplasia & $42(18.8)$ \\
\hline Adenoma, high-grade dysplasia & $4(1.8)$ \\
\hline Cancer cell & $19(8.5)$ \\
\hline Cannot be evaluated & $20(8.9)$ \\
\hline \multicolumn{2}{|l|}{ Endoscopically PCR } \\
\hline PCR & $204(91.5)$ \\
\hline PIR & $19(8.5)$ \\
\hline \multicolumn{2}{|l|}{ Following management } \\
\hline Surveillance endoscopy & $191(85.7)$ \\
\hline Surgery & $32(14.3)$ \\
\hline
\end{tabular}

ECC, early colon cancer; LST, laterally spreading tumor; G-M, granular mixed type; G-H, granular homogenous type; NG-F, non-granular flat type; NG-PD, non-granular pseudo depressed type; EMR, endoscopic mucosal resection; EPMR, endoscopic piecemeal mucosal resection; PCR, presumed complete resection; PIR, presumed incomplete resection.

nite cancer cells were identified in 19 cases (8.5\%). Twenty lesions (8.9\%) did not have evaluable margins because they were torn into multiple pieces during the resection.

\section{Clinical Outcomes after Endoscopic Resection}

Surveillance colonoscopy was performed in 191 cases of 183 patients (85.1\%), 122 cases with negative resection margins, 54 cases with dysplastic cells (including 12 cancer-positive cases), and 15 cases with uncertain resection margins. The mean follow-up period was $51.9 \pm 30.8$ months. Two recurred cancers were detected during the surveillance period. One case of a negative resection margin originally involved a 25 mm granular, mixed, laterally spreading tumor (LST) that was 


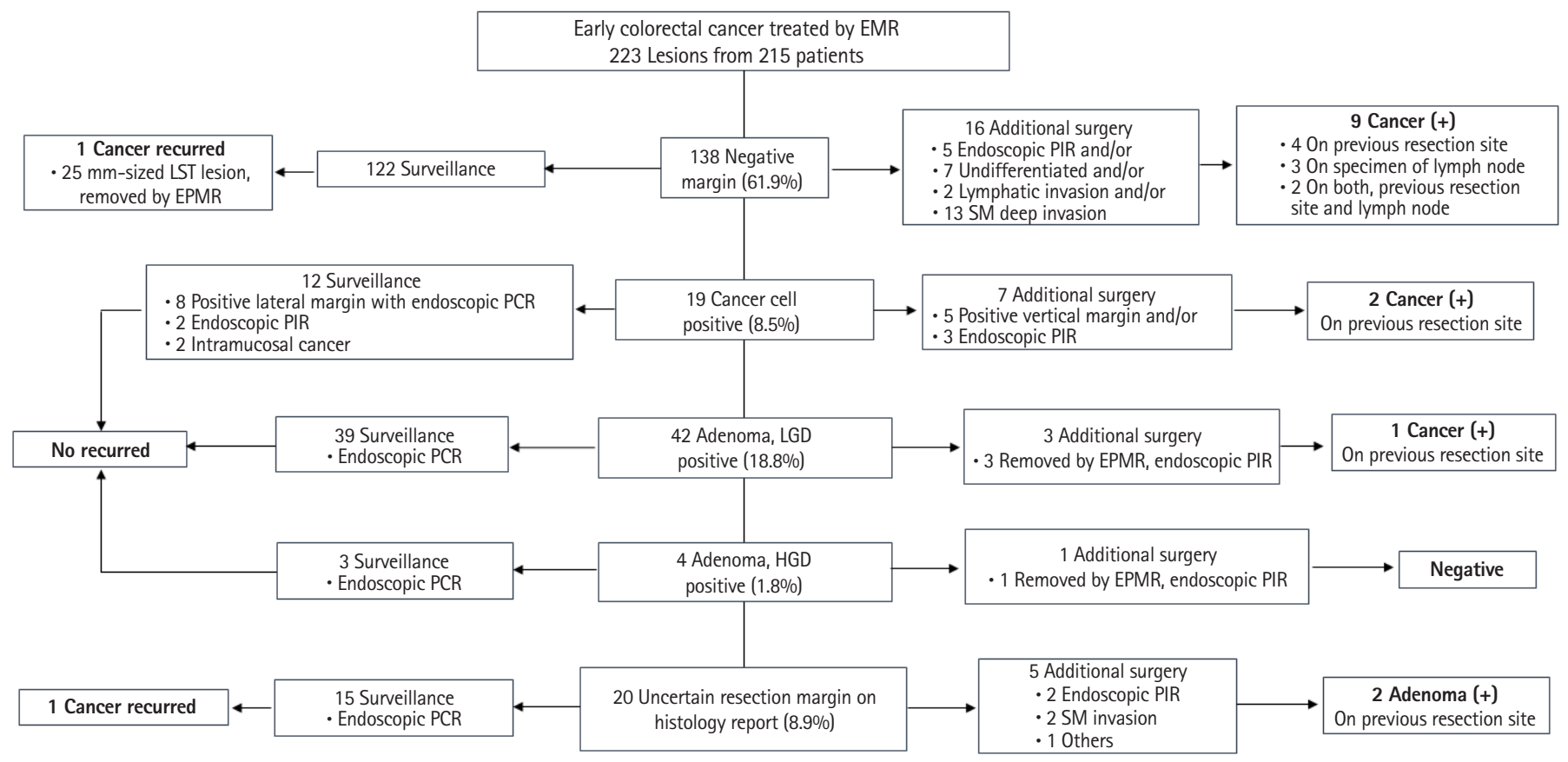

Fig. 2. Study flowchart. Among the 191 lesions that were followed using surveillance colonoscopy with another systemic evaluation, 2 cases of cancer recurrence were identified. Among the 32 lesions that were treated using subsequent surgery, remnant cancers were identified in 12 cases and adenomas were identified in 2 cases. EMR, endoscopic mucosal resection; LGD, low-grade dysplasia; HGD, highgrade dysplasia; LST, lateral spreading tumor; EPMR, endoscopic piecemeal mucosal resection; PCR, presumed complete resection; PIR, presumed incomplete resection; SM, submucosa.

removed by piecemeal EMR. It was a moderately differentiated adenocarcinoma with submucosal invasion $(170 \mu \mathrm{m})$. The histological result revealed complete resection. However, a recurrent non-granular, flat, elevated LST was detected the first endoscopy follow-up 6 months later. The lesions were surgically removed. On pathological examination, the recurrent well-differentiated adenocarcinoma had invaded the SM (700 $\mu \mathrm{m})$, but did not show evidence of lymph node metastasis. The second case of recurred cancer was a $3 \mathrm{~mm}$ depressed lesion with PCR using en bloc EMR. The pathology result was carcinoma in situ without mention of margin status. The endoscopic resection completeness could not be presumed from clinical reports. A recurrent well-differentiated adenocarcinoma was identified as advanced stage during surveillance endoscopy 4 years and 5 months later (Fig. 2). It also showed skin metastasis and was managed with systemic chemotherapy. Subsequent surveillance was performed in 42 other cases (91\%) with an adenoma-positive resection margin and showed no recurrence.

Subsequent surgeries were performed in 32 patients (14.8\%), including the 16 negative margin cases with invasive histological findings (Fig. 2). Residual dysplastic cells were identified in
14 specimens after subsequent surgery, and 12 were cancer cells. The histological results revealed 9 residual cancers at the previous endoscopic resection sites and three cancers in surgically removed lymph nodes. Two cases had cancers at both the resection site and lymph nodes. Three cases of residual cancer were lesions with PIRs. Two cancers were found in cases of cancer-positive resection margin, which were both positive on the lateral and vertical margins. Residual adenomas were detected at the resection sites in 2 of the surgically managed cases among cases of uncertain resection margin. No additional colon cancers developed among the surgically managed patients during a mean follow-up period of $49.2 \pm$ 25.9 months (Fig. 3).

The 2 cases of recurring cancer detected during surveillance and 12 cases of cancer identified histologically at resection sites are evidence of residual cancers following endoscopic resection. Presumed resection completeness, cellular differentiation, and depth of invasion were all characteristics that differed among residual cancers after endoscopic resection of ECC (Table 2). 


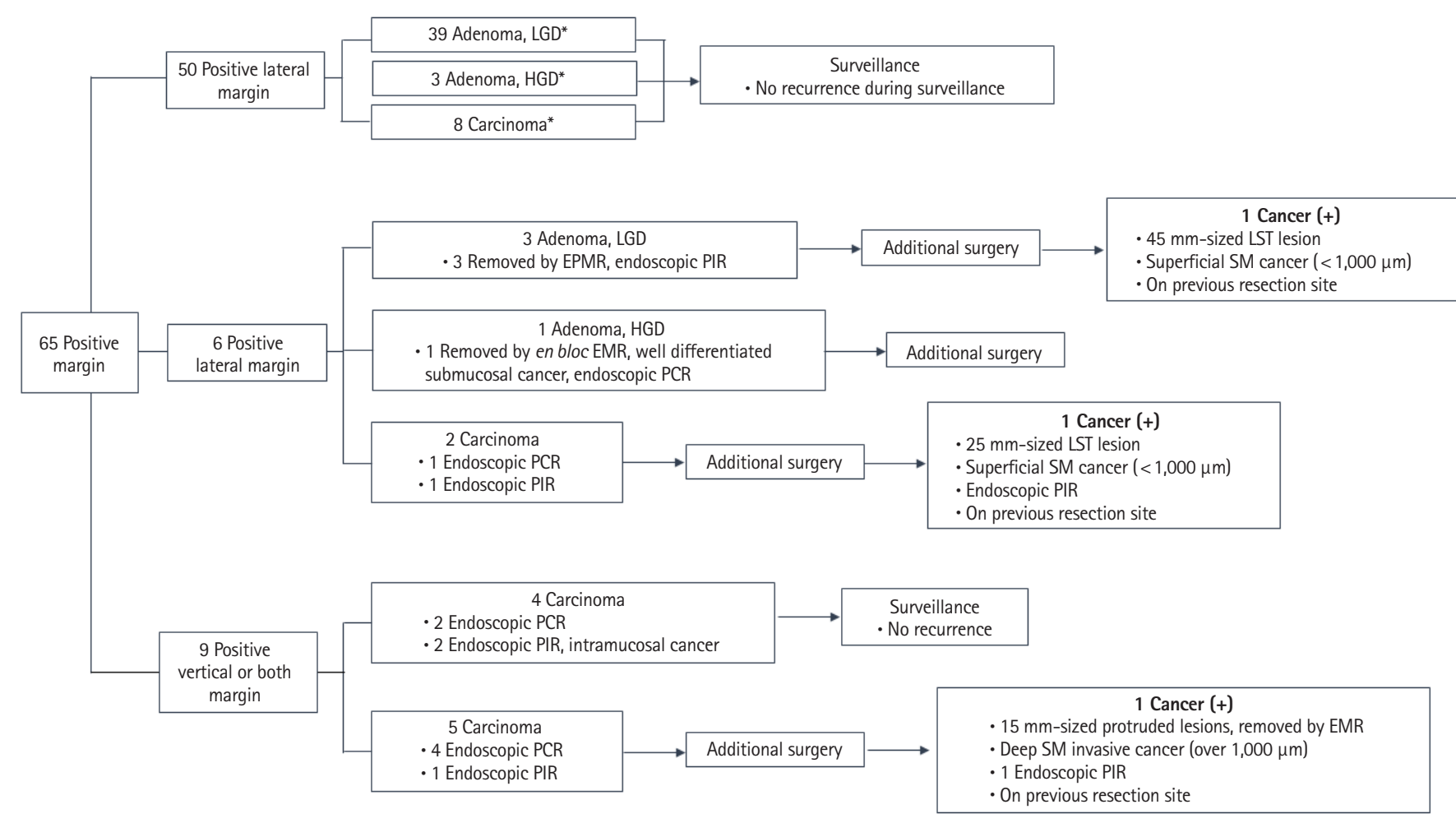

Fig. 3. Study flowchart of 65 cases with positive resection margins. Among the 56 lesions with a positive lateral margin, 50 with endoscopically presumed complete resection (PCR) were followed, and there was no recurrence during surveillance. Three remnant cancers were detected during subsequent surgery of 6 cases of positive lateral margin and 9 cases of positive vertical or both margins. Three residual cancer cases included lesions that underwent endoscopically presumed incomplete/uncertain resection. LGD, low-grade dysplasia; HGD, high-grade dysplasia; EPMR, endoscopic piecemeal mucosal resection; PIR, presumed incomplete resection; EMR, endoscopic mucosal resection; LST, lateral spreading tumor; SM, submucosa.

\section{Clinical Factors Influencing the Choice of Subsequent Surgery}

Logistic regression analyses revealed that subsequent surgery was preferred for PIRs (OR, 15.553; 95\% CI, 4.277-56.558), lesions with SM invasion (OR, 25.459; 95\% CI, 7.090-91.423), and lesions with lymphatic invasion (OR, 13.686; 95\% CI, 1.757106.573) (Table 3).

Subgroup analyses of the 85 lesions, except those with negative resection margin characteristics, revealed that the choice of surgery for subsequent management was independently associated with PIR (OR, 12.735; 95\% CI, 2.138-75.846) and greater invasion depth (OR, 41.348; 95\% CI, 3.182-537.238) (Table 4).

\section{Clinical Factors Related to the Residual Cancers after Endoscopic Resection}

Regardless of method of treatment following EMR, 14 cases of residual cancers were independently associated with PIR (OR, 4.594; 95\% CI, 1.214-17.385) or SM invasion (OR, 5.139; 95\%

\section{CI, 1.182-22.338) (Table 5).}

\section{DISCUSSION}

Endoscopic removal of malignant colonic lesions can prevent colorectal cancer-related mortality. ${ }^{18,19}$ Curative endoscopic resection can be expected for lesions that are confined to the mucosa or the first $1 \mathrm{~mm}$ of the $\mathrm{SM}$, as well as lesions that are well-differentiated, without lymphovascular spread or ulceration, and with a low budding grade. ${ }^{20-24}$ The aim of endoscopic resection is radical excision with negative lateral and vertical margins, and several studies and guidelines recommend targeting a sufficient margin, although the precise distance of the margin from the tumor remains controversial. ${ }^{22,23}$ Moreover, clinicians may not always accept the histological evaluation of the resection margin, as endoscopic resection using electrothermal cautery can produce cytological artifacts. Elongation and hyperchromasia of nuclei are the most common diathermic injuries and can lead to over-interpretation of the 
Table 2. Comparison of Characteristics Grouped by Residual Cancer after Endoscopic Resection

\begin{tabular}{|c|c|c|c|}
\hline Characteristic & No residual cancer $(n=209)$ & Residual cancer $(n=14)$ & $P$-value \\
\hline Sex & & & 0.384 \\
\hline Male & $135(64.6)$ & $8(57.1)$ & \\
\hline Age (yr) & $59.0 \pm 9.2$ & $59.5 \pm 9.1$ & 0.857 \\
\hline Follow-up duration (mon) & $52.6 \pm 31.2$ & $53.9 \pm 18.9$ & 0.818 \\
\hline Right colon & $35(16.7)$ & $4(28.6)$ & \\
\hline Transverse colon & $29(13.9)$ & $2(14.3)$ & \\
\hline Left colon & $74(35.4)$ & $6(42.9)$ & \\
\hline Rectum & $71(34.0)$ & $2(14.3)$ & \\
\hline Size $(\mathrm{mm})$ & $18.8 \pm 15.3$ & $16.9 \pm 10.4$ & 0.635 \\
\hline Resection method & & & 0.171 \\
\hline EMR & $170(82.2)$ & $23(71.9)$ & \\
\hline EPMR & $39(17.8)$ & $9(28.1)$ & \\
\hline Ulcerative lesion & & & $>0.999$ \\
\hline Ulcerative & $2(1.0)$ & 0 & \\
\hline Non-ulcerative & 207 (99.0) & $14(100.0)$ & \\
\hline Lifting on submucosal injection & & & 0.515 \\
\hline Yes & $199(95.2)$ & $13(7.1)$ & \\
\hline Differentiation & & & 0.003 \\
\hline $\mathrm{CIS}$, well differentiation & $168(80.4)$ & $6(42.9)$ & \\
\hline Moderately/poorly differentiation & $41(19.6)$ & $8(57.1)$ & \\
\hline Depth of invasion & & & $<0.0001$ \\
\hline Mucosa & 163 (78.0) & $4(28.6)$ & \\
\hline Submucosa & $46(22.0)$ & $10(71.4)$ & \\
\hline Lymphatic invasion & & & 1.000 \\
\hline Positive & $9(4.3)$ & 0 & \\
\hline Negative & $200(95.7)$ & 14 (100.0) & \\
\hline Resection margin properties & & & 0.339 \\
\hline Cancer & $17(8.1)$ & $2(14.3)$ & \\
\hline Non-cancer (includes negative) & 192 (91.9) & $12(85.7)$ & \\
\hline
\end{tabular}

Values are presented as number (\%) or mean \pm SD.

LST, laterally spreading tumor; EMR, endoscopic mucosal resection; EPMR, endoscopic piecemeal mucosal resection; PCR, presumed complete resection; $\mathrm{PIR}$, presumed incomplete resection; $\mathrm{CIS}$, carcinoma in situ; NA, not available. 
Table 3. Clinical Factors Influencing the Decision to Perform Subsequent Surgery

\begin{tabular}{|c|c|c|}
\hline Clinical factor & Univariate analysis OR (95\% Cl) & Multivariate analysis OR $(95 \% \mathrm{Cl})$ \\
\hline \multicolumn{3}{|l|}{ Endoscopically presumed resection completeness } \\
\hline $\mathrm{PIR} / \mathrm{PCR}$ & $11.982(4.336-33.114)$ & $15.553(4.277-56.558)$ \\
\hline Moderately, poorly differentiated/CIS, well differentiated & $4.074(1.855-8.951)$ & $0.394(0.113-1.380)$ \\
\hline \multicolumn{3}{|l|}{ Depth of invasion } \\
\hline \multicolumn{3}{|l|}{ Lymphatic invasion } \\
\hline Positive/negative & $26.460(5.206-134.494)$ & $13.686(1.757-106.573)$ \\
\hline \multicolumn{3}{|l|}{ Resection margin histological properties } \\
\hline Cancer/non-cancer (includes negative) & $4.177(1.503-11.603)$ & $0.390(0.082-1.860)$ \\
\hline
\end{tabular}

PIR, presumed incomplete resection; PCR, presumed complete resection; CIS, carcinoma in situ.

Table 4. Subgroup Analyses of Subsequent Surgery on Early Colon Cancer Lesions with Positive Resection Margin

\begin{tabular}{|c|c|c|}
\hline Clinical factor & Univariate analysis OR $(95 \% \mathrm{Cl})$ & Multivariate analysis OR $(95 \% \mathrm{Cl})$ \\
\hline \multicolumn{3}{|l|}{ Lifting on submucosal injection } \\
\hline No/yes & $15.692(1.512-162.848)$ & $12.071(0.460-316.798)$ \\
\hline \multicolumn{3}{|l|}{ Endoscopically presumed resection completeness } \\
\hline $\mathrm{PIR} / \mathrm{PCR}$ & $8.167(2.237-29.814)$ & $12.735(2.138-75.846)$ \\
\hline \multicolumn{3}{|l|}{ Differentiation of the cancer } \\
\hline Moderately, poorly differentiated/CIS, well differentiated & $6.667(1.999-22.238)$ & $0.173(0.017-1.805)$ \\
\hline \multicolumn{3}{|l|}{ Depth of invasion } \\
\hline Submucosa/mucosa & $17.024(4.258-68.056)$ & $41.348(3.182-537.238)$ \\
\hline \multicolumn{3}{|l|}{ Lymphatic invasion } \\
\hline Positive/negative & $20.100(3.554-113.690)$ & $10.541(0.827-134.338)$ \\
\hline \multicolumn{3}{|l|}{ Resection margin histologic properties } \\
\hline Cancer/adenoma (LGD+HGD), uncertain & $3.694(1.149-11.874)$ & $0.333(0.039-2.824)$ \\
\hline
\end{tabular}

PIR, presumed incomplete resection; PCR, presumed complete resection; CIS, carcinoma in situ; LGD, low-grade dysplasia; HGD, high-grade dysplasia.

Table 5. Clinical Factors Related to the Residual Cancers after Endoscopic Resection

\begin{tabular}{lcc}
\hline Clinical factor & Univariate analysis OR (95\% Cl) & Multivariate analysis OR (95\% Cl) \\
\hline $\begin{array}{l}\text { Endoscopically presumed resection completeness } \\
\text { PIR/PCR }\end{array}$ & $7.738(2.284-26.219)$ & $4.594(1.214-17.385)$ \\
$\begin{array}{l}\text { Differentiation of the cancer } \\
\text { Moderately, poorly differentiated/CIS, well differentiated }\end{array}$ & $5.463(1.797-16.614)$ & $1.803(0.447-7.283)$ \\
$\begin{array}{l}\text { Depth of invasion } \\
\text { Submucosa/mucosa }\end{array}$ & $8.859(2.655-29.554)$ & 5.139 (1.182-22.338) \\
$\begin{array}{l}\text { Lymphatic invasion } \\
\text { Positive/negative }\end{array}$ & $1.070(1.033-1.109)$ & - \\
Resection margin histological properties & & - \\
Cancer/non-cancer (includes negative) & $4.177(1.503-11.603)$ & \\
\hline
\end{tabular}

PIR, presumed incomplete resection; PCR, presumed complete resection; CIS, carcinoma in situ. 
lesion. ${ }^{25,26}$ In the present study, the retrospective review of endoscopic images and medical records was associated with a level of subjective assessment. However, cases with PIR typically exhibited specific features, including remnant tissue, small tissue islands torn during piecemeal resection, or incomplete coagulation of the tumor.

The present study revealed some discrepancies between the histological results, presumed completeness of the resection, and the outcomes of remnant cancers. For example, a 25mm granular, mixed LST was thought to be completely resected, and the histological results revealed negative margins, although recurrent cancer was discovered within 6 months. A SM cancer with high-grade dysplasia at the margin was managed using subsequent surgery, although no evidence of dysplasia was detected at the resection site. Moreover, 2 cases had cancer-positive margins and PIR, although no evidence of recurrence was detected during follow-up. These discrepancies could be related to differences in opinion between the pathologists and the clinicians who selected the management approach after endoscopic resection. In this study, PIR and SM invasion were important factors when considering subsequent surgery after EMR. PIR and SM invasion were also related to residual cancer cases after endoscopic resection (Table 5). Therefore, PIR and SM invasion were considered in determining the course of treatment management following endoscopic resection and were factors affecting patient outcomes.

Endoscopic PCR may be an important factor in deciding whether to perform additional surgery by confirming removal of remnant cells. Among the 19 cases with cancer-positive resection margins, 12 cases managed with endoscopic surveillance did not develop cancer recurrence. Although there were 2 cases with endoscopic PIR, the remaining 10 cases had intramucosal cancers or were PCR on a lateral margin. Two cases of endoscopic PCR were positive for cancer recurrence during surveillance. The first $25 \mathrm{~mm}$-sized LST cases were removed with piecemeal EMR. Piecemeal resection is generally known to carry risk of recurrence. The resection method may affect clinical outcome but did not show statistical significance in this study. Another case of recurrence was examined via endoscopy 53 months after resection. A relatively long surveillance period was used because recurrence was not detected in early stages.

The decision to treat after endoscopic resection should be based on careful consideration of not only these endoscopic findings, but also the various clinical factors, including the pathologic outcomes and the method of endoscopic resection.
There are no published guidelines on the subsequent management of cases with an adenoma-positive resection margin. Although one cancer was detected after subsequent surgery in the case of an adenoma-positive lateral resection margin removed by piecemeal resection and was PIR, 42 cases with subsequent surveillance have shown no recurrence during the surveillance period.

The present study had several limitations. First, we examined data from 2004 to 2014, and the treatment guidelines for ECC were modified by several professional societies during that period. Thus, differences in the guidelines may have influenced clinical decision-making in many ways, such as choice of the endoscopy device and the method of endoscopic resection. Second, 10 clinicians were involved in patient management during the study period, and their interpretations of the guidelines may have varied, although there were also many agreements. For example, there was a tendency to manage ECC using subsequent surgery if the lesions exhibited deeper invasion or signs suggesting advanced disease, such as surface ulcers, lymphatic invasion, and a "non-lifting" appearance. Thus, the outcomes may not reflect outcomes that can be achieved using the current guidelines.

ECC cases removed by endoscopic submucosal dissection (ESD) methods were not enrolled. ESD is an advanced endoscopic procedure that enables en bloc resection of a lesion. ESD has a low likelihood of removing positive dysplastic cells on the resection margin compared to EMR with piecemeal resection. Thus, ESD cases should be analyzed independently of EMR cases. In addition, the sample size was relatively small, and an extended follow-up is needed to sufficiently evaluate the outcomes of ECC. Thus, we only evaluated 215 patients who underwent follow-up for at least 2 years, although only 2 cases developed a malignancy during the follow-up period, which was too small for subgroup analyses.

The determination of endoscopic PCR can be subjective. Endoscopic PCR or PIR was determined based on the endoscopic images and detail contents of endoscopic reports which the endoscopist recorded whether they presumed complete removal. During our retrospective review of 10 years of data on ECC treated by EMR, we found some cases where we could not determine why subsequent surgery was needed based only on the pathologic EMR report. We saw that the decision regarding subsequent surgery was affected not only by pathologic outcomes but also by the endoscopist's opinion on whether complete resection was obtained, which we called endoscopic presumption in this study. Our results should not be 
used to replace the pathological results from resected lesions, as endoscopic management is becoming possible for less-invasive tumors and earlier stages of disease. Thus, our results to not indicate that the current approach should be modified, although they do highlight potential discrepancies between the clinical and pathological evaluations. Therefore, clinical decision-making should be based on both the pathology report and the presumed completeness of endoscopic resection, as well as the clinician's experience with the indications for endoscopic treatment of ECC and its outcomes.

This study retrospectively evaluated cases with endoscopically resected ECC and revealed that subsequent surgery was associated with SM invasion, lymphatic invasion, poor differentiation, and cancer-positive margins. In addition, presumed completeness of the resection may be helpful for guiding the subsequent management of patients who undergo endoscopic resection of ECC. Careful consideration should be given to follow-up management of endoscopically resected ECC. There may be discrepancies between clinical features and outcomes. They should reflect not only the endoscopic findings but also various clinical factors including the pathologic characteristics and the method of endoscopic resection.

\section{FINANCIAL SUPPORT}

This work was supported by the Soonchunhyang University Research Fund.

\section{CONFLICT OF INTEREST}

No potential conflict of interest relevant to this article was reported.

\section{AUTHOR CONTRIBUTION}

Conceptualization: Kim HG. Methodology: Park J, Kim HG, Jeon SR. Formal analysis: Kim HG, Park J. Project administration: Kim HG, Kim J, Jeon SR, Jeong SO, Cho Y. Visualization: Youn HJ, Ryu S, Song HY. Writing - original draft: Park J, Kim HG. Writing - review and editing: Kim HG, Jin SY, Jeen YM. Approval of final manuscript: all authors.

\section{ORCID}

Park J

Kim HG https://orcid.org/0000-0001-5607-1041

https://orcid.org/0000-0001-7545-4638
Jeong SO

Jo HG

Song HY

Kim J

Ryu S

Cho Y

Youn HJ

Jeon SR

Kim JO

Ko BM

Jeen YM

Jin SY https://orcid.org/0000-0003-3965-6261

https://orcid.org/0000-0002-0947-7185

https://orcid.org/0000-0002-4726-9045 https://orcid.org/0000-0001-5585-5346 https://orcid.org/0000-0001-9715-8514 https://orcid.org/0000-0001-9201-5928 https://orcid.org/0000-0002-7774-7458 https://orcid.org/0000-0001-6970-9737 https://orcid.org/0000-0001-7036-3155 https://orcid.org/0000-0002-0635-4454 https://orcid.org/0000-0003-3608-0279 https://orcid.org/0000-0002-9900-8322

\section{REFERENCES}

1. Okuyama T, Oya M, Ishikawa H. Budding as a risk factor for lymph node metastasis in pT1 or pT2 well-differentiated colorectal adenocarcinoma. Dis Colon Rectum 2002;45:628-634.

2. Yim K, Won DD, Lee IK, Oh ST, Jung ES, Lee SH. Novel predictors for lymph node metastasis in submucosal invasive colorectal carcinoma. World J Gastroenterol 2017;23:5936-5944.

3. Ueno H, Hase K, Hashiguchi Y, et al. Novel risk factors for lymph node metastasis in early invasive colorectal cancer: a multiinstitution pathology review. J Gastroenterol 2014;49:13141323.

4. Bosch SL, Teerenstra S, de Wilt JH, Cunningham C, Nagtegaal ID. Predicting lymph node metastasis in pT1 colorectal cancer: a systematic review of risk factors providing rationale for therapy decisions. Endoscopy 2013;45:827-834.

5. Suh JH, Han KS, Kim BC, et al. Predictors for lymph node metastasis in T1 colorectal cancer. Endoscopy 2012;44:590-595.

6. Beaton C, Twine CP, Williams GL, Radcliffe AG. Systematic review and meta-analysis of histopathological factors influencing the risk of lymph node metastasis in early colorectal cancer. Colorectal Dis 2013;15:788-797.

7. Kitajima K, Fujimori T, Fujii S, et al. Correlations between lymph node metastasis and depth of submucosal invasion in submucosal invasive colorectal carcinoma: a Japanese collaborative study. J Gastroenterol 2004;39:534-543.

8. Cooper HS, Deppisch LM, Gourley WK, et al. Endoscopically removed malignant colorectal polyps: clinicopathologic correlations. Gastroenterology 1995;108:1657-1665.

9. Doornebosch PG, Tollenaar RA, De Graaf EJ. Is the increasing role of Transanal Endoscopic Microsurgery in curation for T1 rectal cancer justified? A systematic review. Acta Oncol 2009; 48:343-353. 
10. Cunningham KN, Mills LR, Schuman BM, Mwakyusa DH. Long-term prognosis of well-differentiated adenocarcinoma in endoscopically removed colorectal adenomas. Dig Dis Sci 1994;39:2034-2037.

11. Volk EE, Goldblum JR, Petras RE, Carey WD, Fazio VW. Management and outcome of patients with invasive carcinoma arising in colorectal polyps. Gastroenterology 1995;109:18011807.

12. Gill MD, Rutter MD, Holtham SJ. Management and short-term outcome of malignant colorectal polyps in the north of England(1). Colorectal Dis 2013;15:169-176.

13. Borschitz T, Gockel I, Kiesslich R, Junginger T. Oncological outcome after local excision of rectal carcinomas. Ann Surg Oncol 2008;15:3101-3108.

14. Bach SP, Hill J, Monson JR, et al. A predictive model for local recurrence after transanal endoscopic microsurgery for rectal cancer. Br J Surg 2009;96:280-290.

15. Shin JW, Han KS, Hyun JH, et al. Risk of recurrence after endoscopic resection of early colorectal cancer with positive margins. Endoscopy 2018;50:241-247.

16. Kim KM, Eo SJ, Shim SG, et al. Risk factors for residual cancer and lymph node metastasis after noncurative endoscopic resection of early colorectal cancer. Dis Colon Rectum 2013;56: $35-42$.

17. Schlemper RJ, Hirata I, Dixon MF. The macroscopic classification of early neoplasia of the digestive tract. Endoscopy 2002; 34:163-168

18. Zauber AG, Winawer SJ, O’Brien MJ, et al. Colonoscopic polyp- ectomy and long-term prevention of colorectal-cancer deaths. N Engl J Med 2012;366:687-696.

19. Lambert R, Kudo SE, Vieth M, et al. Pragmatic classification of superficial neoplastic colorectal lesions. Gastrointest Endosc 2009;70:1182-1199.

20. ASGE Technology Committee, Kantsevoy SV, Adler DG, et al. Endoscopic mucosal resection and endoscopic submucosal dissection. Gastrointest Endosc 2008;68:11-18.

21. Watanabe T, Itabashi M, Shimada Y, et al. Japanese Society for Cancer of the Colon and Rectum (JSCCR) guidelines 2014 for treatment of colorectal cancer. Int J Clin Oncol 2015;20:207239.

22. Bujanda L, Cosme A, Gil I, Arenas-Mirave JI. Malignant colorectal polyps. World J Gastroenterol 2010;16:3103-3111.

23. Butte JM, Tang P, Gonen M, et al. Rate of residual disease after complete endoscopic resection of malignant colonic polyp. Dis Colon Rectum 2012;55:122-127.

24. ASGE Standards of Practice Committee, Fisher DA, Shergill AK, et al. Role of endoscopy in the staging and management of colorectal cancer. Gastrointest Endosc 2013;78:8-12.

25. Goldstein NS, Watts JC, Neill JS, et al. The effect of electrothermal cautery-assisted resection of diminutive colonic polyps on histopathologic diagnosis. Am J Clin Pathol 2001;115:356361.

26. Groisman GM, Amar M, Meir A. Utility of MIB-1 (Ki-67) in evaluating diminutive colorectal polyps with cautery artifact. Arch Pathol Lab Med 2007;131:1089-1093. 